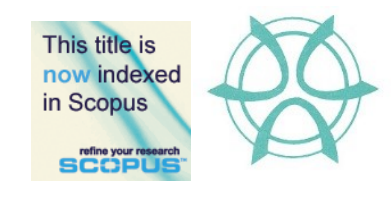

\title{
PROFILE OF AEDES BREEDING IN URBAN HIGH-RISE BUILDINGS: A CASE STUDY OF DENGUE OUTBREAK IN MALACCA, MALAYSIA
}

\author{
Wan Nor Fareeda W. Yahya ${ }^{1}$, Nazri Che Dom ${ }^{2}$ \\ ${ }^{1}$ Centre of Environmental Health and Safety, Faculty of Health Sciences \\ UNIVERSITI TEKNOLOGI MARA \\ ${ }^{2}$ Integrated Mosquito Research Group (I-MeRGe), Faculty of Health Sciences \\ UNIVERSITI TEKNOLOGI MARA
}

\begin{abstract}
An understanding of dengue vector ecology plays an integral role in the effective elimination of vector breeding sites. The aim of this study was to assess the profile of the breeding habitat of the Aedes mosquito in urban high-rise buildings in Malacca. This study utilized epidemiological data from 2013 to 2017 to determine the distribution of dengue cases and Aedes breeding sites in high-rise buildings. The Analytical Hierarchy Process (AHP) was used to evaluate the characteristics of Aedes breeding in high-rise buildings. In addition, the age and the total number of floors in the high-rise buildings were found to be important determinants of DF. The findings from this study will aid in the identification of target areas, which in turn can support future community-based search-anddestroy intervention efforts. Furthermore, the conclusions of this research may guide the future design and construction features of high-rise buildings in Malaysia, which in turn can contribute toward a holistic and sustainable environment in the country.
\end{abstract}

Keywords: Aedes, dengue outbreak, high-rise buildings, Analytical Hierarchy Process (AHP)

\footnotetext{
${ }^{2}$ Associate Professor at Universiti Teknologi MARA Email: nazricd@uitm.edu.my
} 
Wan Nor Fareeda \& Nazri Che Dom

Profile of Aedes Breeding in Urban High-Rise Buildings: A Case Study of Dengue Outbreak in Malacca, Malaysia

\section{INTRODUCTION}

Dengue fever (DF) is an important mosquito-borne viral disease that affects tropical and subtropical regions worldwide, predominantly in urban and semiurban areas. The World Health Organization (WHO) has classified dengue fever as a major public health concern due to the expanding geographical distribution of both the virus and the mosquito vector. DF can be transmitted primarily by the Aedes aegypti (Linnaeus) as well as through the Aedes albopictus (Skuse) (Gubler, 1998). Both species have been found to be responsible for the transmission of the dengue virus in many urban areas in Southeast Asia, including Malaysia. The Aedes mosquito can be found breeding in proximity to human settlements, typically in natural and artificial water-holding containers, and the life-cycle of the vector is closely related to human activities. Among the preferred breeding sites of the vector are ant traps, earthen jars, flower pots, drums, concrete tanks, coconut shells, and discarded tyres (Paupy et al., 2009; Dom et al., 2013a, Dom et al., 2013b). The rise in DF is of concern since there is no curative treatment for the viral disease. At present, abate, an organophosphate larvicide, is widely used for the control of dengue vectors in many parts of the world, including Malaysia.

The abundance of Aedes mosquitoes has had a negative impact on the quality of human life. The survival rate of the mosquitoes and the increase in dengue cases are influenced by several climatic factors. Among the governing parameters are rainfall, humidity, and warm temperatures (Barclay, 2007; Hii et al. 2009), which provide optimal conditions for mosquitoes to breed and expand into new territories. Malaysia has a tropical climate with an average temperature of $27^{\circ} \mathrm{C}$ and an average rainfall of $250 \mathrm{~cm}$. The high temperature, relative humidity and rainfall throughout the year provide ideal conditions for the Aedes mosquito to thrive and breed. Cheong et al. (2014) documented that human settlements and non-agricultural areas largely influence the incidence of DF. Aedes mosquitoes have been found indoors and outdoors in human settlements, especially in culverts, water compartments, metal drums, plastic drums, pails, gully traps, discarded containers, and solid waste dumps, open spaces, construction sites, and factories. Furthermore, it is claimed that buildings with flawed structural designs contribute to the transmission of dengue (Omonikweinka \& Iyagba, 2005; Yee, 2014). This study was aimed at further investigating and analysing the research gap in the identification of Aedes breeding sites on building structures. The findings will be useful in guiding relevant policy and public health decision makers in tackling the root causes of dengue outbreaks in Malaysia and other countries with similar climate characteristics. Community empowerment in identifying breeding places is a key aspect in the prevention and control of dengue. The conclusions of this study will help to highlight potential areas of concern with the goal of strengthening the overall dengue prevention strategy framework. 
PLANNING MALAYSIA

Journal of the Malaysia Institute of Planners (2020)

\section{RESEARCH BACKGROUND}

\section{Study Population}

The Melaka Tengah district was selected as the main research site to study the distribution of Aedes breeding habitats in urban high-rise buildings. Melaka Tengah, with an area of $314 \mathrm{~km}^{2}$, is the capital of the state of Malacca. It is comprised of 44 towns and 95 villages, the total population of which was more than 503,000 residents in 2016, of whom 303,000 were Malays (58\%), 169,000 were Chinese (32\%), 22,000 were Indians $(4 \%)$, and the rest were of other ethnicities. This district is administrated by two local authorities, namely, Majlis Bandaraya Melaka Bersejarah (MBMB) and Majlis Perbandaran Hang Tuah Jaya (MPHTJ) (Figure 1).

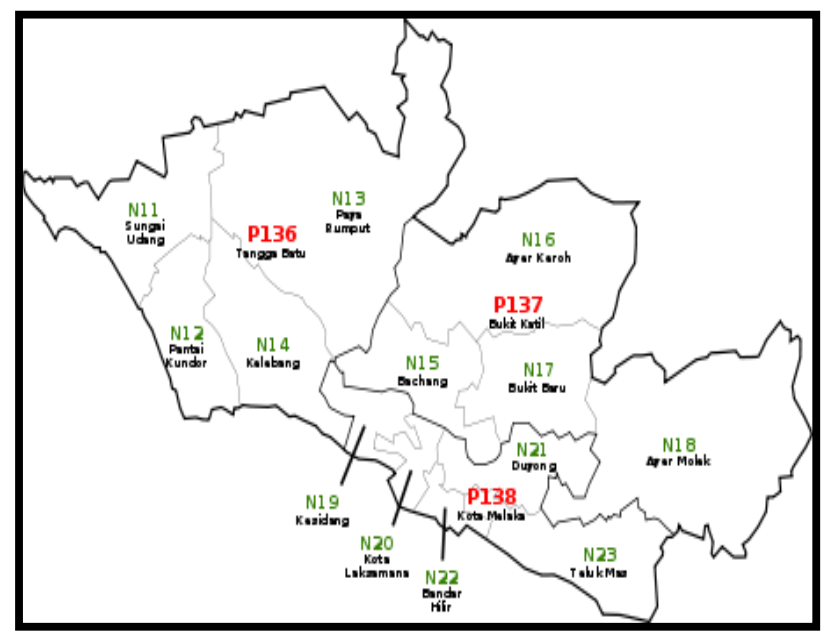

Figure 1: Administrate areas in Melaka Tengah. The red and green colours indicate the Parliament zone and DUN, respectively.

\section{Study design}

This study applied an integrated observational epidemiological approach to determine the distribution of dengue cases and outbreaks as well as the types of containers and the main problems in high-rise buildings. Data from January 2013 to December 2017 were extracted from the e-Dengue version 2.0 online system of the Vector-Borne Disease Control Sector of the Ministry of Health (MOH). The study design required "exposure information" for the complete profile of the breeding habitats of the Aedes mosquito in areas with dengue fever outbreaks from 2013 - 2017. The research methodology consisted of three phases, namely, (i) distribution pattern of DF in high-rise buildings, (ii) profile of Aedes breeding habitats in urban high-rise buildings, and (iii) evaluation of the main problems in high-rise buildings using AHP. 
Wan Nor Fareeda \& Nazri Che Dom

Profile of Aedes Breeding in Urban High-Rise Buildings: A Case Study of Dengue Outbreak in Malacca, Malaysia

\section{Data collection and management}

The proposal for this study was submitted to the National Medical Research Register (NMRR) (NMRR-17-3503-38568 (IIR) for ethical clearance. This research utilized dengue surveillance data (DSD) collected from the Vector Control Division, Malacca State Health Department. The original DSD is a dailybased dataset compiled between 2013 and 2017 with a few attributes (year, month, type of container, mean AI, mean BI, mean CI, mean PI). The dataset consists of relevant information concerning the breeding habitats of the Aedes mosquito in high-rise buildings in Melaka Tengah from 2013 - 2017.

The preliminary phase of this study involved the determination of the distribution pattern of dengue outbreaks in high-rise buildings in the Melaka Tengah District from 2013 - 2017. In relation to the risk and exposure to DF, high-density population settlements in Melaka Tengah are associated with escalating incidences of DF cases. Moreover, the number of DF cases in Melaka Tengah is amongst the highest in Malacca compared to other districts. Even though the Melaka Tengah district has undergone extensive development, this study assessed the differences in the risk of exposure to DF and the distribution of outbreaks in specific premises in high-rise buildings between 2013 and 2017.

The aim of this study was to identify the distribution pattern of dengue outbreaks in high-rise residential buildings in the Melaka Tengah district, Malacca from 2013 to 2017. The data represented secondary data retrieved from the $\mathrm{MOH}$. The cases were categorised into two groups; either single cases or outbreaks. The data were computed according to outbreak categories, namely, controlled, uncontrollable, and hotspot outbreaks. Only the data on the outbreaks were maintained for further analysis. The epidemiological data were aimed at determining the dengue risk areas in Melaka Tengah, while the mosquito breeding habitat data were aimed at demonstrating the major contributing factors to dengue outbreaks, and the breeding container data at correlating the source of the issues. The distribution and pattern of dengue outbreaks involving high-rise residential buildings in Melaka Tengah were selected for the study.

Two types of data were analysed, namely, the data on mosquito breeding habitats and types of breeding containers. This study integrated the environmental indicators and the demographic profile of the dengue vector distribution for the surveillance, monitoring and evaluation of dengue outbreaks. These in turn were correlated with the types of containers used for breeding, the locations of the containers (indoor/outdoor), the Aedes index (AI), Breteau index (BI) and Container index (CI) for the outbreaks, and the classification of the mosquito larvae species within residential areas to ensure that the type of larva belonged to the Aedes mosquito. The AI, BI and CI are commonly used to record Aedes mosquito infestation levels, and to evaluate the distribution and density of the mosquito species in a study area. 


\section{RESULTS AND DISCUSSION}

Figure 2 illustrates the temporal pattern of DF cases in Melaka Tengah from 2013 - 2017. The number of DF cases increased in 2013, especially in the middle of that year, but gradually declined. Incidents surged in the early months of 2014 before fluctuating, and the highest peak was reached in 2015. Following this, the number of DF cases reported steadily declined from 2016-2017. The temporal pattern of DF cases in Melaka Tengah from 2013 - 2017 was subjected to further analysis. The results were divided into five fractions, namely I, II, III, IV and V, to represent the years 2013, 2014, 2015, 2016 and 2017, respectively. In order to determine the pattern of DF cases for each year, a simple linear regression approach was used to estimate the general pattern of DF cases in response to the months of the year. The plot of the monthly DF cases revealed increasing trends in the number of DF cases reported, the number of DF cases in outbreaks, and the number of DF cases reported in high-rise buildings in the Melaka Tengah district from 2013 to 2017. The number of DF cases due to outbreaks escalated significantly between the years 2014-2015 in high-rise buildings.

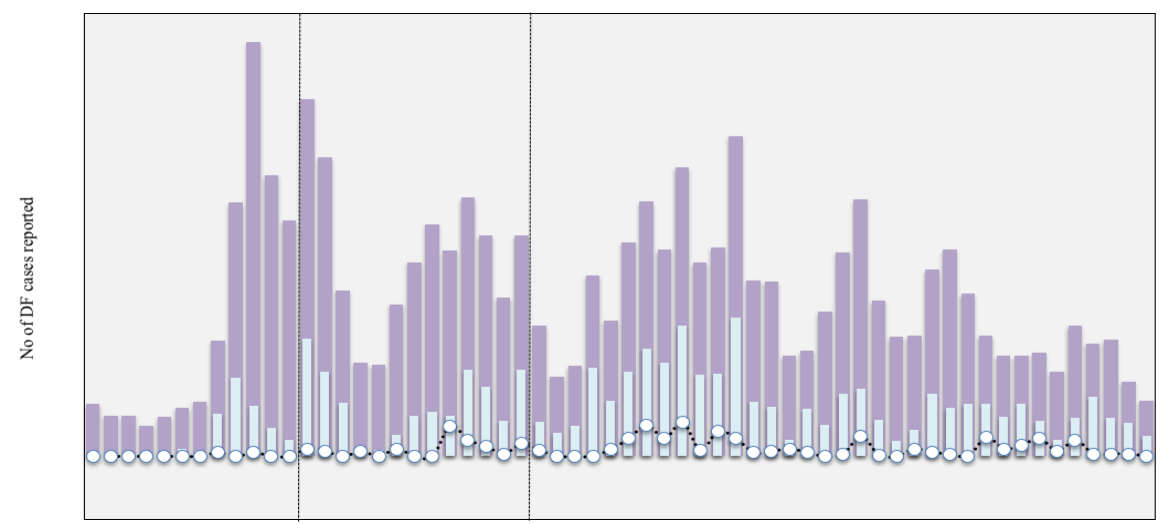

Figure 2: Temporal pattern of DF cases in Melaka Tengah from 2013 to 2017

All the data on DF outbreaks were categorized according to the outbreak status, namely; (i) controlled outbreaks, (ii) uncontrollable outbreaks, and (iii) hotspot outbreaks. Table 1 represents the total number of dengue outbreaks from 2013 to 2017 , of which $12.5 \%$ of them were reported in high-rise residential buildings. The outbreak cases were classified as follows: 57 controlled outbreaks, 2 uncontrollable outbreaks, and 4 hotspot outbreaks. 
Wan Nor Fareeda \& Nazri Che Dom

Profile of Aedes Breeding in Urban High-Rise Buildings: A Case Study of Dengue Outbreak in Malacca, Malaysia

Table 1: Distribution pattern of dengue outbreaks in Melaka Tengah district from 2013 to 2017 based on the status of the outbreak

\begin{tabular}{|c|c|c|c|c|c|c|}
\hline \multirow{2}{*}{ Years } & \multirow{2}{*}{$\begin{array}{l}\text { Total of } \\
\text { Dengue } \\
\text { Outbreaks }\end{array}$} & \multirow{2}{*}{$\begin{array}{l}\text { Total Outbreak } \\
\text { Involve High } \\
\text { Rise Residential } \\
\text { Building }\end{array}$} & \multirow{2}{*}{ Percent } & \multicolumn{3}{|c|}{$\begin{array}{l}\text { Classification of outbreaks } \\
\text { in high-rise buildings }\end{array}$} \\
\hline & & & & C1 & $\mathbf{C 2}$ & Hotspot \\
\hline 2013 & 37 & 2 & $5 \%$ & 2 & & \\
\hline 2014 & 101 & 11 & $11 \%$ & 11 & & \\
\hline 2015 & 149 & 18 & $12 \%$ & 14 & 1 & 3 \\
\hline 2016 & 120 & 16 & $13 \%$ & 16 & & \\
\hline 2017 & 100 & 16 & $16 \%$ & 14 & 1 & 1 \\
\hline Total & 507 & 63 & $12.5 \%$ & 57 & 2 & 4 \\
\hline
\end{tabular}

Note: Control outbreak (C1) refers to locality with successfully of prevention action and end after 14 days. Uncontrollable outbreak (C2) refers to locality when there are occurred a new case after 14 days and Hotspot refers to locality reaches 30 days and beyond the day of the outbreak started (MOH, 2008).

Next, a total of 63 dengue outbreaks in Melaka Tengah were further analysed. Overall, $92 \%(58 / 63)$ of the outbreaks occurred in areas where there were positive mosquito breeding sites on their premises. $47.6 \%(30 / 63)$ of the outbreaks were positive for the Aedes albopictus, and 11.1\% (7/63) for the Aedes aegypti. 33.3\% (21/63) of the mosquitoes detected were of mixed breed (Aedes aegypti with Aedes albopictus), a finding that was comparable with previous reports. Table 2 highlights the risk categorizations of the outbreaks according to the mosquito indices. Out of the 63 outbreaks that were analysed, 202 of the breeding containers were positive with Aedes species. The controlled outbreaks revealed the highest number of positive breeding containers, with $32 \%(65 / 202)$ of the containers being found indoors, and 52\% (106/202) outdoors in high-rise buildings. $3 \%(6 / 202)$ of the breeding containers were found to have mosquitoes breeding only in outdoor areas for the uncontrollable outbreaks. Positive breeding containers were also found in indoor and outdoor areas for hotspots, but at very low frequencies, accounting for 4\% (8/202) and 9\% (17/202), respectively. 
PLANNING MALAYSIA

Journal of the Malaysia Institute of Planners (2020)

Table 2: Risk categorizations of outbreaks according to mosquito indices

\begin{tabular}{|c|c|c|c|c|c|}
\hline \multicolumn{3}{|c|}{ Status of Outbreak } & Control & Uncontrollable & Hotspot \\
\hline \multirow{2}{*}{\multicolumn{2}{|c|}{$\begin{array}{l}\text { Breeding container } \\
\qquad(\mathbf{n}=\mathbf{2 0 2})\end{array}$}} & $\begin{array}{c}\text { Indoor } \\
(\%)\end{array}$ & $65(32)$ & 0 & $8(4)$ \\
\hline & & $\begin{array}{c}\text { Outdoor } \\
(\%)\end{array}$ & $106(52)$ & $6(3)$ & $17(9)$ \\
\hline \multirow{12}{*}{ 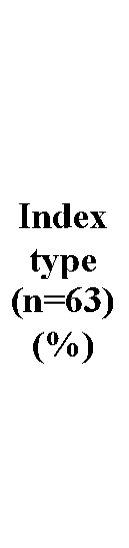 } & Premise & $<1$ & $24(38)$ & $1(2)$ & $4(6)$ \\
\hline & Index & $1-5$ & $32(50)$ & $1(2)$ & 0 \\
\hline & (PI) & $>5$ & $1(2)$ & 0 & 0 \\
\hline & Aedes & $<1$ & $34(54)$ & $2(3)$ & $4(6)$ \\
\hline & Index & $1-4$ & $22(35)$ & 0 & 0 \\
\hline & $(\mathrm{AI})$ & $>4$ & $1(2)$ & 0 & 0 \\
\hline & Breteau & $<1$ & $20(32)$ & $2(3)$ & $1(2)$ \\
\hline & Index & $1-4$ & $37(58)$ & 0 & $3(5)$ \\
\hline & $(\mathrm{BI})$ & $>4$ & 0 & 0 & 0 \\
\hline & \multirow{3}{*}{$\begin{array}{l}\text { Container } \\
\text { Index }(\mathrm{CI})\end{array}$} & $<1$ & $50(79)$ & $2(3)$ & $4(6)$ \\
\hline & & $1-10$ & $6(10)$ & 0 & 0 \\
\hline & & $>10$ & $1(2)$ & 0 & 0 \\
\hline
\end{tabular}

The controlled outbreaks, with a range of 1 to 5 , recorded the highest PI $(50 \% ; 32 / 63)$ compared to the uncontrollable outbreaks $(2 \% ; 1 / 63)$, in the range $<1$ and 1 to 5 , and the hotspot outbreaks $(6 \% ; 4 / 63)$ in the range of $<1$. For the outbreak status, the controlled outbreaks reported a high AI in the range of between 1 and $4(54 \%)$ and $>4(35 \%)$. This signified that most of the houses had positive breeding sites. None of the outbreaks had a BI of $>4$, and the controlled outbreak had a high BI value in the range 1 to $4(58 \%$; 37/63). However, several outbreaks revealed values of $<1$. A total of $88 \%(56 / 63)$ of the outbreaks had low levels of transmission based on the CI, $10 \%(6 / 63)$ of the areas had a moderate CI, and 2\% (1/63) had a high CI. The mixed breeding of Aedes albopictus and Aedes aegypti was detected in containers from the controlled and hotspot outbreak areas.

In order to evaluate the problem of the breeding of the Aedes mosquito in high-rise residential buildings, five factors were identified and clustered according to their domain of influence, namely; (i) building structure, (ii) age of building, (iii) maintenance status, (iv) total number of residences, and (v) total number of floors. All the criteria were assigned different ratings on a scale of 1 (low relationship with dengue outbreak) to 7 (high relationship with dengue outbreak), according to a comprehensive analysis of the local data obtained by different analyses. The results obtained from a preliminary analysis of all the factor groups and criteria after evaluation are summarized in Figure 3. The most 
Wan Nor Fareeda \& Nazri Che Dom

Profile of Aedes Breeding in Urban High-Rise Buildings: A Case Study of Dengue Outbreak in Malacca, Malaysia

significant factors were building structure, age of building, maintenance status, total number of residences, and total number of floors. Most of the problems were found to be related to the age of building $(\mathrm{CR}=0.07)$ and the total number of floors $(\mathrm{CR}=0.07)$. The results indicated that each factor had its own role in enhancing the transmission of dengue. The $C R$ values were lower than 0.1 , which indicated that the use of weights was suitable. The problems in high-rise buildings were derived by following the weighted linear combination method.

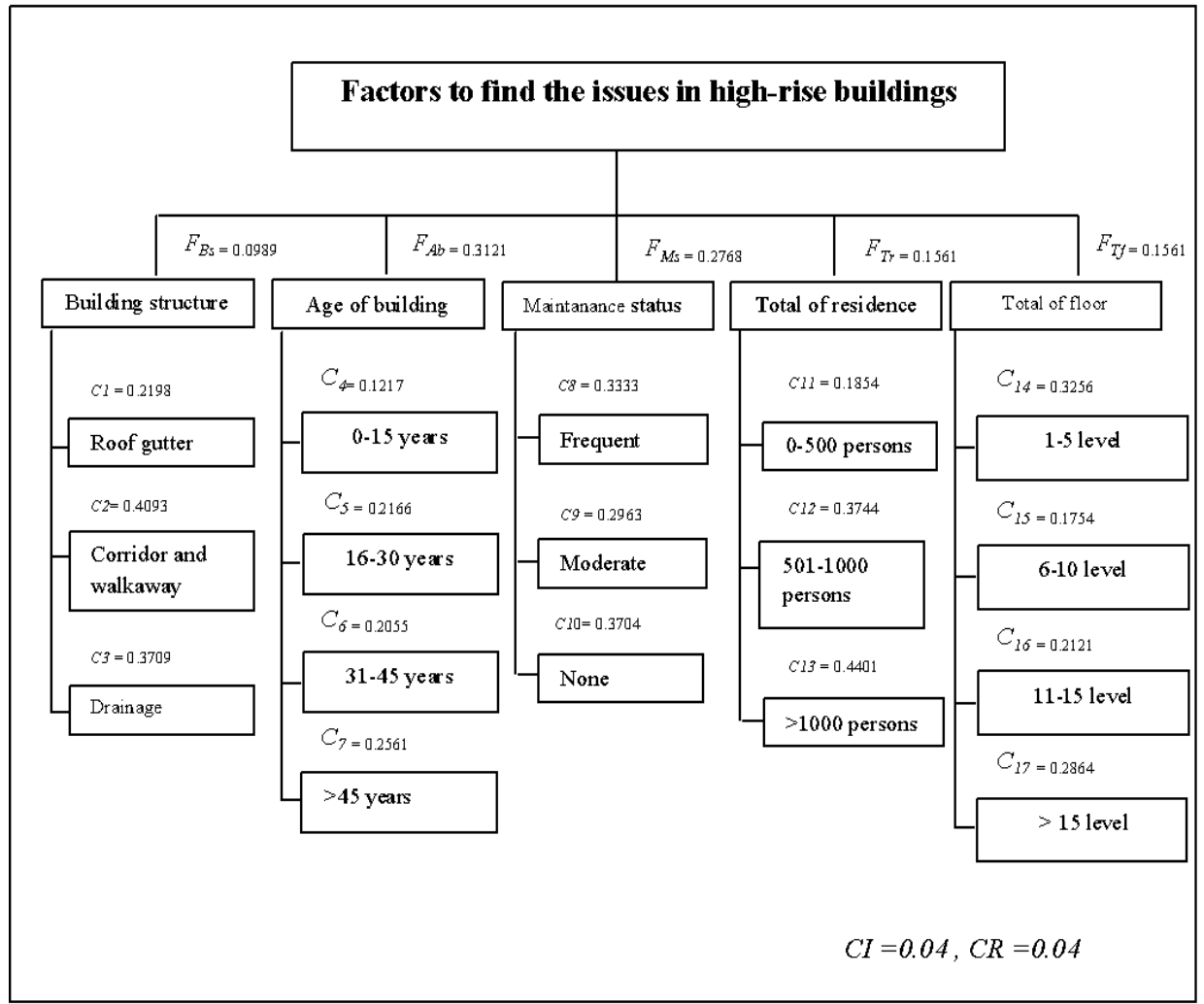

Figure 3: Weights of all the decision processes. The symbol $(\mathrm{F})$ indicates the main criteria, and $(\mathrm{C})$ indicates the sub-criteria.

The nature of the vector-breeding habitat is associated with the influence of the building design on the human social factor. The design of the high-rise buildings affects the assessment of certain residences. The body tasked with the management of high-rise buildings has failed in certain areas, including the lack of a septic tank management system, abandoned rain gutters, and poor air-well management. This was proven in the findings, where the public and the building management failed to achieve a satisfactory level of hygiene awareness. The behaviour and habits of people living in high-rise buildings, regardless of 
their economic status, are still traditional when it comes to managing municipal waste. Despite a regular waste management collection schedule, the presence of illegal settlements is still evident. On the other hand, the results also revealed that kitchen utensils and other man-made containers were left uncovered outside. Malaysia, being a tropical country, experiences frequent rainfall throughout the year. Water tends to stagnate in the exposed containers, thereby providing breeding sites for the Aedes mosquito. The breeding sites point to the failure of the building management to systematically maintain gully traps and rooftops. These findings indicate that the building design and surrounding environment will affect the breeding habitat of the Aedes mosquito. DF outbreaks can be permanently controlled by improving the environmental conditions. The dengue transmission chain can be broken by destroying the habitats of the Aedes mosquito and halting the life cycle of the immature mosquitoes. Measures to prevent Aedes infestation can be made feasible by simply removing the stagnant water, which can be effectively achieved through public campaigns and vector control activities. The relationship between density and risk will vary temporarily, depending on factors like human social factors, population growth, uncontrolled and underserviced urbanization, and the deterioration of high-rise building infrastructures.

The key to effectively controlling DF outbreaks is to recognize highpotential transmission areas with the densest human and vector populations. The results of the present study demonstrated significant differences in the preferred breeding habitats, and highlighted potential areas of concern, which can form the baseline information in detecting the potential occurrence of DF outbreaks, especially involving high-rise buildings. The findings from this study provide insights that could help policymakers to formulate guidelines aimed at tackling the root causes of the escalating dengue crisis in Malaysia and neighbouring countries. In addition, such findings can give rise to greater community empowerment and enhanced social responsibility to aid in the identification, evaluation and elimination of breeding places, thereby helping to augment and strengthen the existing dengue prevention framework.

\section{ACKNOWLEDGEMENTS}

The contribution of research funding from Research Management Centre (RMC) Universiti Teknologi MARA (UITM), Universiti Teknologi MARA (UiTM) and Ministry of Higher Education (MOHE) Malaysia are also duly acknowledged.

\section{REFERENCES}

Achee N.L, Gould F, Perkins T.A, Reiner R.C Jr, Morrison A.C, Ritchie S.A, (2015). A Critical Assessment of Vector Control for Dengue Prevention. PLoS Neglected Tropical Disease 9 (5); e0003655 
Wan Nor Fareeda \& Nazri Che Dom

Profile of Aedes Breeding in Urban High-Rise Buildings: A Case Study of Dengue Outbreak in Malacca, Malaysia

Aidil Roslan, Aziz Shafie, Romano Ngui, Yvonne Ai L.L, Wan Yusoff W.S (2013). Vertical Infestation of the Dengue Vectors Aedes Aegypti And Aedes albopictus In Apartments In Kuala Lumpur, Malaysia. Journal of the American Mosquito Control Association, 29(4):000-000

Cheong, Y.L., Leitao, P.J. \& Lakes, T. (2014). Assessment of land uses factors associated with dengue cases in Malaysia using Boosted Regression Trees. Spatial and Spatio-temporal Epidemiology, (10),75-84.

Dom, N. C., Ahmad, A. H., Ishak, A. R., \& Ismail, R. (2013a). Assessing the risk of dengue fever based on the epidemiological, environmental and entomological variables. Procedia-Social and Behavioral Sciences, (105),183-194.

Dom, N. C., Ahmad, A. H., \& Ismail, R. (2013b). Habitat characterization of Aedes sp. breeding in urban hotspot area. Procedia-Social and Behavioral Sciences, (85), 100-109.

Gubler, D.J (1998). Resurgent Vector-Borne Diseases as a Global Health Problem. Emerging Infectious Diseases, 4(3), 442-450

Hii, Y.L., Rocklöv, J., Ng, N., Tang, C.S., Pang, F.Y. \& Sauerborn, R. (2009). Climate variability and increase in intensity and magnitude of dengue incidence in Singapore. Global Health Action, (2).

Monica Danial , Subramaniam S., Yoon C.K, Ong L.M (2016). External Factors Governing Dengue Outbreaks and Practices Associated in Curbing Dengue Infections among Population in Northern Malaysia International Journal of Health Sciences \& Research (www.ijhsr.org). 6 (5).

Nazri Che Dom, A. Abu Hassan, Z. Abd Latif and Rodziah Ismail (2011). Colloquium on Humanities, Science and Engineering Research. Impact of Climate and Land Use Variability Based on Dengue Epidemic Outbreak in Subang Jaya, 907 - 912. doi:978-1-4673-0020

Omonikweinka, R.R. \& Iyagba, A. (2005). The Menace of Sick Buildings: A Challenge to All For Its Prevention and Treatment. Lagos: University of Lagos Press.

Paupy, C., Delatte, H., Bagny, L., Corbel, V., \& Fontenille, D. (2009). Aedes albopictus, an arbovirus vector: from the darkness to the light. Microbes and Infection, 11(14), 1177-1185.

Wan-Norafikah, O, Nazni, W.A., Noramiza, S, Shafa'ar-Ko'ohar, S. Azirol-Hisham, A., Nor-Hafizah, R., Sumarni, M.G., Mohd-Hasrul, H., Sofian-Azirun, M. and Lee, H.L (2012). Distribution of Aedes Mosquitoes in Three Selected Localities in Malaysia. Sains Malaysiana 41(10): 1309-1313

Wan-Norafikah, O, Nazni, W.A., Noramiza, S, Shafa'ar-Ko'ohar, S., Azirol-Hisham, A., Nor-Hafizah, R., Sumarni, M.G., Mohd-Hasrul, H., Sofian-Azirun, M. and Lee, H.L (2010). Vertical dispersal of Aedes (Stegomyia) spp. in high-rise apartments in Putrajaya, Malaysia. Tropical Biomedicine 27(3): 662-667

Yee, F.P. (2014). Authorities need to get to root cause of dengue fever problem. The Star, Kuala Lumpur.

Received: January 2020. Accepted: $13^{\text {th }}$ May 2020 